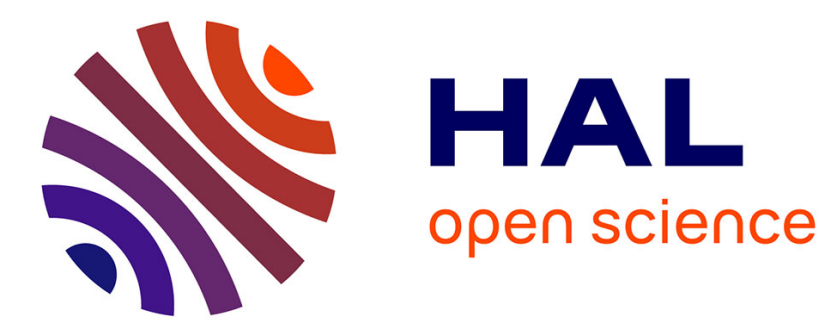

\title{
Françoise Lavocat, Les personnages rêvent aussi \\ Ninon Chavoz
}

\section{To cite this version:}

Ninon Chavoz. Françoise Lavocat, Les personnages rêvent aussi. Lectures, 2021, 10.4000/lectures.47646. hal-03468165

\section{HAL Id: hal-03468165 \\ https://hal.science/hal-03468165}

Submitted on 6 Dec 2021

HAL is a multi-disciplinary open access archive for the deposit and dissemination of scientific research documents, whether they are published or not. The documents may come from teaching and research institutions in France or abroad, or from public or private research centers.
L'archive ouverte pluridisciplinaire HAL, est destinée au dépôt et à la diffusion de documents scientifiques de niveau recherche, publiés ou non, émanant des établissements d'enseignement et de recherche français ou étrangers, des laboratoires publics ou privés. 


\section{Françoise Lavocat, Les personnages rêvent aussi}

\section{Ninon Chavoz}

\section{(2) OpenEdition \\ Journals}

\section{Electronic version}

URL: https://journals.openedition.org/lectures/47646

DOI: $10.4000 /$ lectures.47646

ISSN: 2116-5289

\section{Publisher}

Centre Max Weber

Brought to you by Université de Strasbourg

\section{이맛제 Les bibliothéques}

de I' Université de Strasbourg

\section{Electronic reference}

Ninon Chavoz, "Françoise Lavocat, Les personnages rêvent aussi", Lectures [Online], Reviews, Online since 14 February 2021, connection on 29 August 2021. URL: http://journals.openedition.org/lectures/ 47646 ; DOI: https://doi.org/10.4000/lectures.47646

This text was automatically generated on 29 August 2021.

(c) Lectures - Toute reproduction interdite sans autorisation explicite de la rédaction / Any replication is submitted to the authorization of the editors 


\title{
Françoise Lavocat, Les personnages rêvent aussi
}

\author{
Ninon Chavoz
}

1 Dans un contexte politiquement, socialement et intellectuellement marqué par la remise en cause de toutes les lignes de démarcation, qu'elles soient nationales ou catégorielles ${ }^{1}$, les études littéraires pourraient bien être tentées de prendre en marche le train des sciences humaines et de réfuter à leur tour l'une des distinctions les plus fondamentales de leur champ d'investigation : celle qui sépare le fait de la fiction. Tout en mettant en garde contre la tentation d'un tel alignement des études littéraires sur les sciences politiques et sociales, Françoise Lavocat reconnaissait dès 2016 tout ce qu'une prise de position en faveur de la frontière avait d'inconfortable: une telle démarche reviendrait en effet à se comporter en "ségrégationnistes ", ou pire encore, en « gardiens rébarbatifs [...] d'un pays peu accueillant à l'égard de la diversité et du métissage $»^{2}$.

2 Ce nouvel essai est l'occasion de prendre cette métaphore au pied de la lettre et de lui conférer ainsi une puissance redoublée: dans la lignée des auteurs du XVII et du XVIII ${ }^{\mathrm{e}}$ siècle, auxquels elle rend un hommage appuyé, Françoise Lavocat invente un pays de Romancie, ou plutôt une planète Fiction où vivent en bonne entente tous les personnages forgés par l'imagination humaine. Le programme intellectuel de la collection «Fictions pensantes » dirigée par Franck Salaün trouve là une saisissante réalisation : c'est en effet en se prêtant à l'exercice jubilatoire de la «théorie-fiction » que l'auteure pense les enjeux de l'abolition de la frontière entre le réel et l'imaginaire. Dotés d'une agentivité autant que d'une réflexivité propres, les personnages permettent ici le déploiement d'une histoire littéraire renouvelée, à moins qu'il ne s'agisse, conformément au projet formulé par Françoise Lavocat dans le cadre de sa délégation à l'Institut Universitaire de France, d'une « démographie des personnages " ${ }^{3}$, qui restitue les évolutions, les migrations et les métamorphoses de ces cohortes fictionnelles. Précisons d'emblée que celles-ci frappent le lecteur par leur éclectisme, qui rend indispensable l'annexe répertoriant leurs origines et leurs biographies : parmi les habitants de la planète Fiction, on compte aussi bien des héros de romans classiques 
que des personnages issus de la littérature populaire, de la bande-dessinée, du cinéma, de l'univers des mangas et de la chanson (comme en témoigne la discrète arrivée de Mr. Tambourine Man, à la suite de l'octroi du prix Nobel de littérature à Bob Dylan, p. 131). La Fiction n'est pas sectaire, c'est là son moindre défaut : ainsi Maigret perce-til à jour les secrets les mieux gardés de la Princesse de Clèves (chapitre XIII), tandis que le balzacien Vautrin, accusé de crime contre la fiction, est mis au pilori par le plaidoyer d'Hermione Granger, héroïne de la saga Harry Potter, transformée en brillante avocate de la partie civile (chapitre XIV). L'essai convie donc les représentants patentés de la grande littérature aux côtés des timides ressortissants des " contre-littératures ", pour reprendre la notion forgée dès 1975 par Bernard Mouralis ${ }^{4}$.

S'il ne dépend pas de la surveillance sourcilleuse d'austères gardiens du temple, garants de la pureté du canon, le droit de résider dans l'une des cités cosmopolites de la planète Fiction - Shadavar, la capitale, est issue du folklore persan, tandis que les villes de Tokoloshe et de Qilin émanent respectivement de la mythologie zouloue et chinoise n'en demeure pas moins soumis à une double condition, garante du respect de la «frontière ». La première est d'être doté d'un « degré de fictionnalité » suffisamment élevé, défini par des « jurys ontologiques » dont le verdict prend appui sur le diagnostic infaillible posé par un "Chiméramètre » (chapitre II). La seconde est de n'avoir pas sombré dans l'oubli des lecteurs, spectateurs et autres consommateurs de fiction: les personnages dont la mémoire n'est plus conservée perdent peu à peu consistance, jusqu'à se transformer en fantômes et à disparaître. C'est pourquoi Vautrin s'inquiète du triste sort imparti aux milliers de personnages balzaciens réunis dans la banlieue d'Honoria : faute d'une action vigoureuse qui autoriserait leur résurrection, ils risquent l'évanouissement définitif, voire «l'extinction de masse » (p. 183). C'est pour pallier ce danger que l'interlope forçat fomente un complot dont l'instrument décisif est une machine à «ondes lectorales » et l'objectif essentiel le renouvellement du lien entre fiction et réalité.

Il est vrai que l'un et l'autre pôle ont connu des transformations substantielles, dont les dialogues à bâtons rompus des personnages s'emploient brillamment à rendre compte : tandis que la première recule, à mesure que les auteurs contemporains donnent le primat au document et au témoignage, la seconde n'a de cesse de « s'augmenter » et de s'enrichir de fake news en tous genres. Deux cas d'école nourrissent ainsi les espoirs les plus fous chez Vautrin et de ses complices. Le premier est celui d'un personnage mineur des Misérables de Victor Hugo, Enjolras : quoiqu'il ne joue pas un rôle essentiel dans le roman, il bénéficie grâce au succès du jeu vidéo de combat japonais Arm Joe d'une aura que lui envient ses collègues. Le sauvetage des personnages par leur conversion ludique est ainsi envisagé, jusqu'à ce qu'une poignée d'avatars désorientés échoue dans la clinique du docteur Ross, tout droit sorti de la série Urgences. La conclusion que consigne ce dernier dans son journal de bord est sans appel: "Les personnages font rêver, penser, permettent de s'identifier, que sais-je. Les avatars servent essentiellement à jouer, même lorsqu'ils ressemblent à des personnages " (p. 178). Le second cas est celui de l'homme politique ukrainien Volodimir Zelensky, volontiers présenté comme un être de fiction parce qu'il incarna un président à l'écran avant d'en assumer réellement la fonction en 2019: il n'en faut pas plus pour que Vautrin et ses acolytes, convaincus que « les personnages sont souvent plus courageux et plus sensés que les humains »(p.155), imaginent Jean Valjean Président des Français. 
5 S'ils nourrissent leurs espoirs les plus effrénés, les lecteurs peuvent pourtant aussi susciter l'ire des créatures de fiction. Comment ne pas leur tenir grief d'avoir condamné sans nuances les œuvres d'un auteur ou d'un réalisateur que ses opinions politiques ou ses exactions supposées vouent aux gémonies de l'opinion (Woody Allen, par exemple, dont le film La Rose pourpre du Caire offre à l'essai de Françoise Lavocat l'une de ses lignes directrices)? Les personnages sont plus scandalisés encore lorsqu'une interprétation hasardeuse des textes d'origine vient porter atteinte à leur intégrité supposée. La vindicte des habitants de Shadavar s'attache ainsi au metteur en scène fictif Herman Sororis, coupable d'avoir donné des grands opéras du répertoire occidental des versions par trop sujettes à ce que Laurent Dubreuil appelle «la dictature des identités » ${ }^{5}$. Inspiré peut-être par l'affaire du « Kanata » de Robert Lepage, dont les représentations durent être annulées faute d'avoir donné la parole à des comédiens amérindiens, Sororis caresse le projet d'une mise en scène de Turandot située non en Chine, mais chez les Indiens Arumbayas imaginés par Hergé dans L'Oreille Cassée, espérant ainsi ne pas "donner lieu à une accusation de racisme». Quant au personnage de Liu, la jeune esclave chinoise amoureuse du prince Calaf, il serait incarné par un contre-ténor travesti. Et l'auteure de conclure, parodiant avec

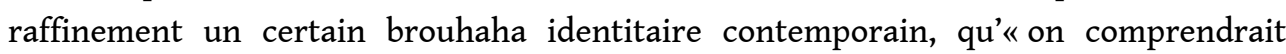
aussi, en voyant à la fin de l'opéra Calaf-Tintin se réveiller sur un misérable grabat dans une rue de Mexico, que cette sinistre rêverie lui aurait révélé à lui-même son homosexualité. Son chant d'amour triomphal, à la fin de la pièce, pourrait alors être interprété comme l'adoption résolue d'une identité enfin acceptée » (p. 61).

Trancher les riches débats contradictoires que suscitent ces cas d'espèce parmi les habitants de Shadavar n'a rien d'aisé : on ne peut que plaindre l'aimable Mr Pickwick chargé du gouvernement de cette tumultueuse assemblée qu'agite sans fin le " démon de la théorie $»^{6}$. Au terme de ce voyage en terres de Romancie, un constat s'impose cependant: s'il est loisible et même excitant d'imaginer des franchissements de la frontière entre réalité et fiction, son abolition n'aboutirait pas nécessairement à une extension du champ des possibles et nous priverait, à tout le moins, de l'incomparable plaisir de la transgression. N'oublions pas (le présent ouvrage en est le meilleur gage !) que les théoriciens rêvent aussi - non plus, comme habitants de Shadavar, de «moutons des pastorales » et de lecteurs dociles, peu au fait des prérogatives que leur confère la fameuse antienne de la reader's response - mais d'amours anachroniques (quelle belle romance que celle de Cecilia, héroïne de La Rose pourpre du Caire et du prince Far-Féredin, tout droit issu du roman contre les romans signé par GuillaumeHyacinthe Bougeant en 1735 !), de «cercles carrés » (chapitre XVI) et de «forêts interprétatives » (chapitre $\mathrm{X}$ ) où persistent d'obscures inscriptions...

\section{NOTES}

1. Voir par exemple à ce propos Dominique Schnapper, L'Esprit démocratique des lois, Paris, Gallimard, 2014.

2. Françoise Lavocat, Fait et fiction : pour une frontière, Paris, Éditions du Seuil, 2016, p. 12. 
3. Voir à ce propos le projet de Françoise Lavocat sur le site de l'Institut universitaire de France : https://www.iufrance.fr/les-membres-de-liuf/membre/508-francoise-lavocat.html, consulté le 5 février 2021.

4. Bernard Mouralis, Les contre-littératures [1975], Paris, Hermann, collection «Fictions Pensantes ", 2011, avec une préface d'Anthony Mangeon.

5. Laurent Dubreuil, La dictature des identités, Paris, Gallimard, 2019.

6. Antoine Compagnon, Le démon de la théorie : littérature et sens commun, Paris, Éditions du Seuil, 1998.

\section{AUTHOR}

\section{NINON CHAVOZ}

Ninon Chavoz est assistante de recherche à l'Université de Mayence. Elle est l'auteur d'une thèse consacrée à la tentation encyclopédique dans l'espace francophone africain et d'un essai, Éloge des ratés. Huit portraits de l'auteur francophone en encyclopédiste, paru en 2020 aux éditions Hermann. 